\title{
Zum internationalen Komplettanbieter herangewachsen
}

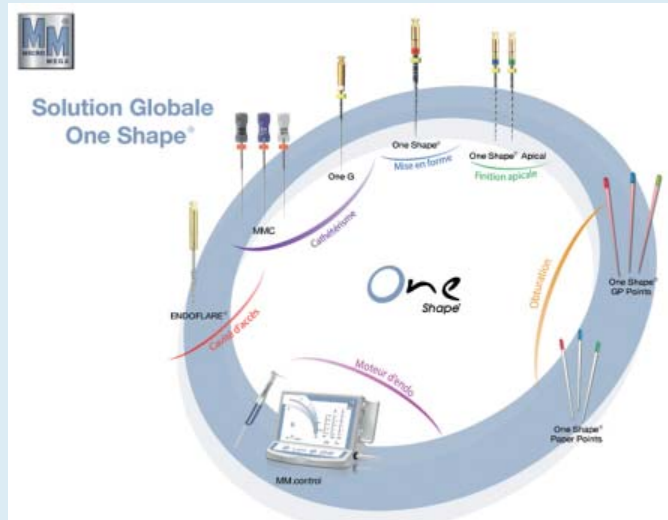

Die Sanavis Group unter der Führung der VDDI-Vorsitzenden Dr. Martin Rickert und Stefan Helsing hat im 5. Jahr ihres Bestehens die Integration abgeschlossen. „Sanavis Group ist zu einem international aufgestellten Komplettanbieter für Endodontie, zahnärztliche Antriebstechnik und dentale Wiederaufbereitung herangewachsen“, sagte Dr. Rickert auf der IDSPressekonferenz der Unternehmensgrup- pe. Unter dem Dach Sanavis haben sich 2011 die Firmen Micro-Mega (Frankreich), SciCan (Kanada) und SycoTec (Deutschland) zu einer strategischen und organisatorischen Allianz zusammengeschlossen. Jedes der 3 Unternehmen wird nach wie vor eigenständig geführt, eine gemeinsame Strategie, gemeinsame Finanzen sowie Kooperationen in den Bereichen Produktion und Innovation, Marketing und Vertrieb sowie internationale Expansion machen es aber möglich, in Märkte vorzudringen, die für jedes einzelne der 3 Unternehmen ansonsten unerreichbar wären.

Als Weltpremiere präsentiert beispielsweise MICRO-MEGA bei der IDS 2015 One $\mathrm{G}$, die sterile NiTi-Feile zum Einmal- gebrauch für die Schaffung eines Gleitpfads in kontinuierlicher Rotation. Mit One G - als Ergänzung zu One Shape wird nur 1 Instrument zur Schaffung eines Gleitpfads benötigt. Ferner stellte das Unternehmen das One Shape Procedure Pack vor. Mit dem NiTi-Instrument Endoflare zur koronalen Erweiterung, den MMCHandfeilen Nr. 10 und 15 sowie den One G- und One Shape New Generation-Feilen als sterile Instrumente zum Einmalgebrauch vereinfacht das One Shape Procedure Pack die tägliche Arbeit des Zahnarzts. Ein klinischer Fall wird mit einem One Shape Procedure Pack behandelt. Die Procedure Packs sind gebrauchsfertig (sterile Instrumente) und werden nicht wiederaufbereitet - Produktvorteile, die eine echte Vereinfachung der endodontischen Behandlung und eine reelle Produktivitätssteigerung bedeuten!

Weitere Informationen erhalten Sie bei MICRO-MEGA, FR - 25006 BESANCON, +33(0)3 81544201 oder info.de@micromega.com

Nach einer Pressemitteilung der Sanavis Group, Leutkirch Internet: www.sanavisgroup.com 